\title{
手性芳氧苯氧丙酰胺类化合物的合成及除草活性研究
}

\author{
杨 森 ${ }^{a}$ 丁成荣 ${ }^{a}$ 刘幸海 ${ }^{a}$ 翁建全 ${ }^{a}$ 袁 静*,b 谭成侠*, $a$ \\ ( ${ }^{a}$ 浙江工业大学化学工程学院 杭州 310014) \\ ( ${ }^{b}$ 浙江农林大学林业与生物技术学院 杭州 311300)
}

\begin{abstract}
摘要 为了寻找高除草活性的农药先导化合物, 通过活性基团拼接原理和生物酶拆分技术, 设计合成了一系列未见文 献报道的具有手性的芳氧苯氧丙酰胺类化合物，其结构经 ${ }^{1} \mathrm{H}$ NMR 和 HRMS 进行了确证. 初步的生物活性测试结果表 明, 目标化合物均对单子叶禾本科杂草显示出较高的选择性除草活性. 在剂量为 $150 \mathrm{~g} / \mathrm{ha}$ 时, 目标化合物对罔草 (Backmannia syzigachne)、棒头草(Polypogon fugax)、早熟禾(Poa acroleuca) 的除草活性均可达 $75 \%$ 以上，其中 3 个化合 物对棒头草(Polypogon fugax)的防除效果达 $100 \%$.
\end{abstract}

关键词 芳氧苯氧丙酸类化合物; 除草剂; 手性; 除草活性; 合成; 酶拆分

\section{Synthesis and Herbicidal Activity of Chiral Aryloxyphenoxypropionic Amides Compounds}

\author{
Yang, Sen ${ }^{a}$ \\ Ding, Chengrong ${ }^{a}$ \\ Liu, Xinghai ${ }^{a}$ \\ Weng, Jianquan ${ }^{a}$ \\ Yuan, Jing*,b \\ Tan, Chengxia*,a \\ $\left({ }^{a}\right.$ College of Chemical Engineering, Zhejiang University of Technology, Hangzhou 310014) \\ ( ${ }^{b}$ College of Forestry and Biotechnology, Zhejiang Agriculture and Forestry University, Hangzhou 311300)
}

\begin{abstract}
In order to find pesticidal lead compounds with high herbicidal activity, a series of novel chiral aryloxyphenoxypropionic amides were designed and synthesized using the principle of active substructure combination and the technology of biological enzyme splitting. The structures of the target compounds were confirmed by ${ }^{1} \mathrm{H} N M R$ and HRMS. The preliminary bioassay data showed that all target compounds displayed excellent herbicidal activity and selectivity against monocotyledonous weeds. At the dosage of $150 \mathrm{~g} / \mathrm{ha}$, the target compounds showed herbicidal activity against Backmannia syzigachne, Polypogon fugax and Poa acroleuca with more than $75 \%$. And the control effects of three compounds against Polypogon fugax Nees were $100 \%$.
\end{abstract}

Keywords aryloxyphenoxypropionic acids; herbicide; chirality; herbicidal activity; synthesize; enzyme splitting

含氮杂环化合物, 如吡啶、嘧啶、噁唑、苯并吡嗪 等, 在农药创制过程中起着重要的作用, 具有广泛的杀 菌 ${ }^{[1 \sim 4]}$ 、除草 ${ }^{[5 \sim 7]}$ 、杀虫 ${ }^{[8 \sim 12]}$ 和调节植物生长 ${ }^{[13]}$ 等活性, 其与苯氧丙酸酯结构组成的芳氧苯氧丙酸酯类化合物 具有较好的除草活性而成为除草剂的研究热点之一(图 1). 芳氧苯氧丙酸酯类(APP)除草剂是一类选择性除草 剂, 通过抑制禾本科植物体内乙酰辅酶 $\mathrm{A}$ 羧化酶的活性 来抑制脂肪酸的合成破坏膜结构而达到除草效果 ${ }^{[14,15]}$.

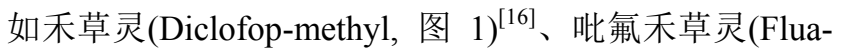
zifop-butyl, 图 1) ${ }^{[17]} 、$ 腎唑禾草灵(Fenoxaprop-p-ethyl, 图
1) ${ }^{[18]}$ 等. 另外, 酰胺类化合物表现出广泛的杀虫 ${ }^{[19-23] 、}$

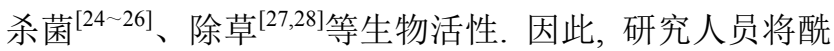
胺活性基团引入到芳氧苯氧丙酸酯类除草剂中来获得 新除草活性化合物, 如 Moon 等 ${ }^{[29]}$ 合成出的一系列取代 苯酰胺类 APP 化合物 A(图 1)可用于水稻田防除禾本科 杂草, $Y u$ 等 ${ }^{[30]}$ 合成出的化合物 B (图 1)表现出良好的除 草活性，在测试浓度为 $100 \mathrm{~g} / \mathrm{L}$ 时，化合物 B 对野油菜的 防除效果为 $87.8 \%$. 研究中发现, 手性结构使农药在药 效、环境安全性等方面具有独特的优势, 能够有效降低 生产成本和减少除草剂施用量 ${ }^{[31 ~ 33]}$. 鉴于此, 为了寻找

* Corresponding authors. E-mail: tanchengxia@zjut.edu.cn; 1015251265@qq.com Received May 13, 2019; revised June 3, 2019; published online June 19, 2019. Project supported by the National Key R \& D Program (No. 2017YFD0200507). 国家重点研发计划(No. 2017YFD0200507)资助项目. 
高除草活性的芳氧苯氧丙酰胺类化合物, 以炔草酯 ${ }^{[34]}$ 和啞唑酰草胺 ${ }^{[35]}$ 为先导化合物, 依据活性基团拼接原 理, 在芳氧苯氧丙酸类结构基础上引入酰胺结构, 并采 取了生物酶拆分技术, 设计并合成了一系列未见报道的 $R$ 构型芳氧苯氧丙酰胺类化合物(图 2), 以期发现具有更 高除草活性的农药先导化合物. 另外, 目标化合物经 ${ }^{1} \mathrm{H}$ NMR 和 HRMS 进行了结构确证, 并进行了除草活性研 究, 结果发现所有目标化合物对单子叶禾本科杂草均具 有良好的除草活性. 目标化合物的合成路线如 Scheme 1 所示.<smiles>COC(=O)C(C)Oc1ccc(Oc2ccc(Cl)cc2Cl)cc1</smiles>

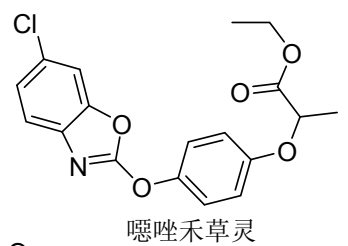<smiles>CCCCOC(=O)C(C)Oc1ccc(Oc2ccc(C(F)(F)F)cn2)cc1</smiles><smiles>[X]c1cc([N+](=O)[O-])ccc1F</smiles>

A<smiles>[B]c1ccc(OCC(=O)NC(c2ccccc2Cl)P(=O)(O)OCC)cc1</smiles>

图 1 禾草灵、吡氟禾草灵、噁唑禾草灵和化合物 $\mathbf{A} 、 \mathbf{B}$ 的化 学结构式

Figure 1 Chemical structures of clodinafop-propargyl, fluazifop-butyl, fenoxaprop-p-ethyl, and compounds A and B

\section{1 结果与讨论}

\section{1 目标化合物的合成}

目标化合物 8 的合成需先以 2-氯丙酸甲酯 1 和对苯 二酚为原料, 甲醇为溶剂, 经醚化反应生成 2-(4-差基苯 氧基)丙酸甲酯(2), 2-(4-羟基苯氧基)丙酸甲酯再经生物 酶米曲霉 WZ007(Aspergillus oryzae WZ007) ${ }^{[36 ~ 38]}$ 进行 拆分, 生成关键中间体( $R$ )-2-(-4-差基苯氧基)丙酸甲酯 (3), 然后水解生成 $(R)-2$-(4-着基苯氧基)丙酸(6), 化合物 6 再与 2,3-二氟-5-氯吡啶进行 Williamson 醚化反应生成 (R)-2-\{4-[(5-氯-3-氟吡啶-2-基)氧基]苯氧基 $\}$ 丙酸(7), 最 后通过活性基团拼接原理与取代苯胺生成目标化合物 8.

在合成关键中间体 $\mathbf{3}$ 的过程中, 采用了生物酶拆分 技术, 而所采用的米曲霉 WZ007 是脂肪酶的一种, 是由
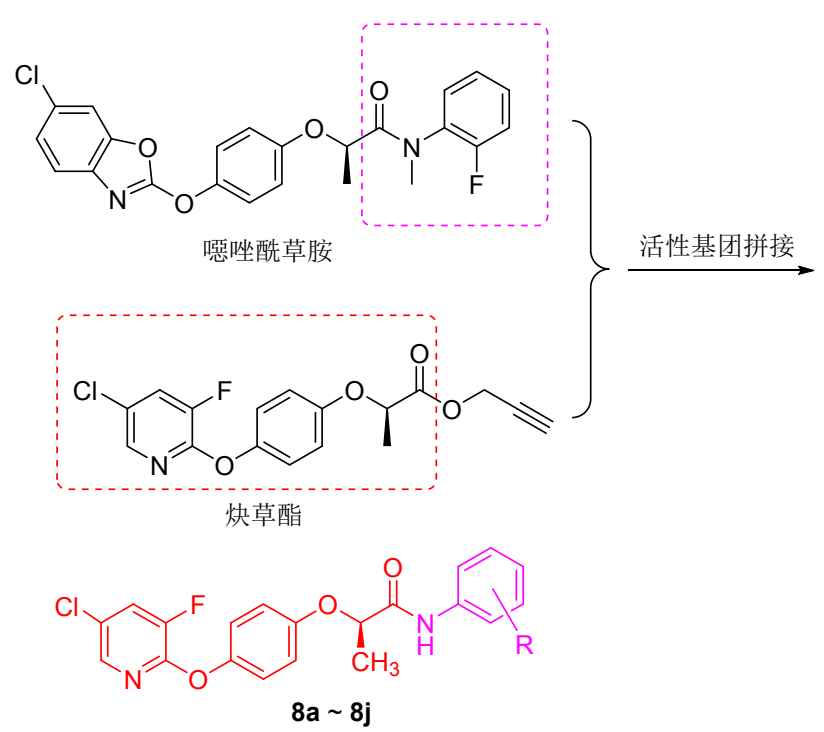

图 2 目标化合物 $\mathbf{8}$ 的设计策略

Figure 2 Design strategy of target compounds 8

浙江工业大学生物工程学院郑建永博士课题组通过富 集培养从土壤中笁选出来. 根据酶的最佳活性温度和最 佳活性 $\mathrm{pH}$, 化合物 2 在 $35{ }^{\circ} \mathrm{C} 、 \mathrm{pH}$ 为 7 的反应条件下 经米曲霉 WZ007 拆分生成关键中间体 $(R)$-2-(-4-差基苯 氧基)丙酸甲酯(3), 经手性液相色谱分析得中间体 $\mathbf{3}$ 的 ee 值可达 98.4\%. 同时也生成了副产物 $(S)-2$-(4-差筇基苯 氧基)丙酸(4), 化合物 4 再与甲醇经酯化反应生成 (S)-2-(4-着基苯氧基)丙酸甲酯，然后在甲苯和甲醇体系 下进行外消旋化反应生成 $(R, S)$-2-(4-羟基苯氧基)丙酸 (5), 化合物 5 再经酯化可生成化合物 2 用于拆分, 以形 成副产物的重复利用.

\section{2 化合物的波谱分析}

以目标化合物 $\mathbf{8 a}$ 为例进行谱学分析, ${ }^{1} \mathrm{H}$ NMR 谱图 中 $\delta 10.15$ 处的单峰为酰胺基氢的吸收峰, $\delta 8.18 \sim 7.01$ 间的多重峰为吡啶环和苯环上氢吸收峰, $\delta 4.88$ 处的四 重峰为叔碳上氢的吸收峰, $\delta 1.57$ 处的双重峰为甲基的 吸收峰; 在 HRMS 谱图中，该化合物分子离子峰计算值 为 $[\mathrm{M}+\mathrm{H}]^{+}$387.0906, 测定值 $[\mathrm{M}+\mathrm{H}]^{+}$387.0902, 绝对 误差在 0.003 以内.

\section{3 化合物的除草活性}

目标化合物的除草活性测试结果见表 1 . 从表 1 可 以看出, 目标化合物 8 在 $150 \mathrm{~g} / \mathrm{ha}$ 剂量下茎叶处理对单 子叶禾本科杂草(茥草、棒头草、早熟禾)均具有良好的 除草活性, 而对双子叶杂草(芥菜、小藜、酸模)无除草 活性, 说明了目标化合物能够选择性的防除单子叶禾本 科杂草. 目标化合物 8 对茥草的抑制活性均在 $80 \%$ 以上， 其中 8c, 8f, 8g, 8h 达到了 $90 \%$; 对早熟禾的除草活性在 $75 \% \sim 80 \%$ 之间; 对棒头草的除草活性分别为 $100 \%$, 


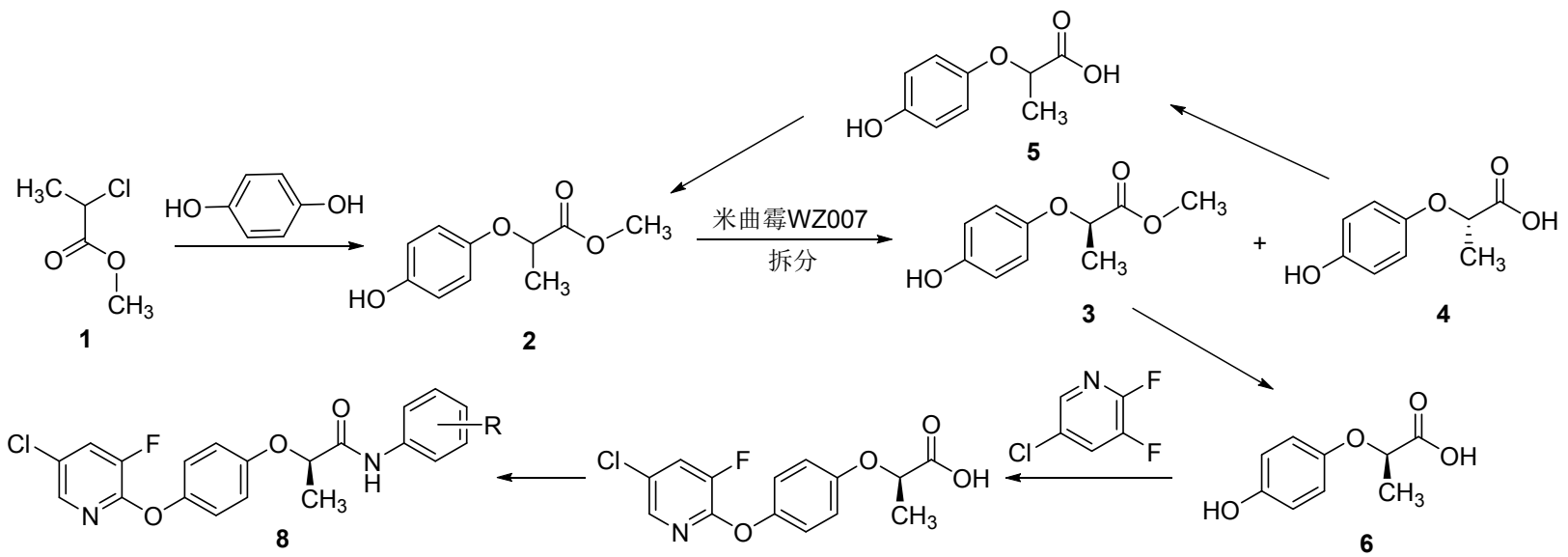

8a: $\mathrm{R}=\mathrm{H}, \mathbf{8 b}=2-\mathrm{OCH}_{3}, \mathbf{8 c}: \mathrm{R}=4-\mathrm{C}\left(\mathrm{CH}_{3}\right)_{3}, \mathbf{8 d}: \mathrm{R}=2,4-\left(\mathrm{CH}_{3}\right)_{2}$,

7

8e: $R=2-\mathrm{CH}_{3}-3-\mathrm{Cl}, 8 \mathrm{f}: \mathrm{R}=2-\mathrm{F}, \mathbf{8 g}: \mathrm{R}=4-\mathrm{F}, 8 \mathrm{~h}: \mathrm{R}=2,6-\mathrm{F}_{2}$

8i: $\mathrm{R}=3-\mathrm{Cl}, 8 \mathrm{j}: \mathrm{R}=4-\mathrm{Cl}$

图式 1 目标化合物 $\mathbf{8}$ 的合成路线

Scheme 1 Synthetic route of target compound $\mathbf{8}$

表 1 在剂量 $150 \mathrm{~g} / \mathrm{ha}$ 下目标化合物 $8 \mathbf{a} \sim \mathbf{8 j}$ 的除草活性(抑制 率/\%)

Table 1 Herbicidal activities (percent inhibition/\%) of title compounds $\mathbf{8 a} \sim \mathbf{8 j}$

\begin{tabular}{ccccccc}
\hline 化合物 & 芥菜 & 小藜 & 酸模 & 䓟草 & 棒头草 & 早熟禾 \\
\hline $\mathbf{8 a}$ & 0 & 0 & 0 & $85 \pm 2$ & $100 \pm 0$ & $80 \pm 1$ \\
$\mathbf{8 b}$ & 0 & 0 & 0 & $85 \pm 3$ & $90 \pm 1$ & $75 \pm 1$ \\
$\mathbf{8 c}$ & 0 & 0 & 0 & $90 \pm 0$ & $90 \pm 4$ & $75 \pm 0$ \\
$\mathbf{8 d}$ & 0 & 0 & 0 & $80 \pm 1$ & $90 \pm 2$ & $80 \pm 0$ \\
$\mathbf{8 e}$ & 0 & 0 & 0 & $85 \pm 0$ & $95 \pm 0$ & $80 \pm 0$ \\
$\mathbf{8 f}$ & 0 & 0 & 0 & $90 \pm 2$ & $90 \pm 1$ & $75 \pm 0$ \\
$\mathbf{8 g}$ & 0 & 0 & 0 & $90 \pm 0$ & $95 \pm 1$ & $80 \pm 0$ \\
$\mathbf{8 h}$ & 0 & 0 & 0 & $90 \pm 1$ & $90 \pm 3$ & $80 \pm 2$ \\
$\mathbf{8 i}$ & 0 & 0 & 0 & $85 \pm 1$ & $100 \pm 0$ & $85 \pm 2$ \\
$\mathbf{8 j}$ & 0 & 0 & 0 & $85 \pm 0$ & $100 \pm 0$ & $80 \pm 1$
\end{tabular}

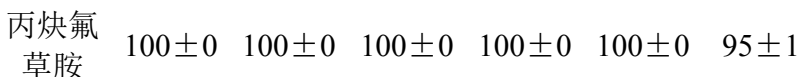

$90 \%, 90 \%, 90 \%, 95 \%, 90 \%, 95 \%, 90 \%, 100 \%, 100 \%$, 可 以看出目标化合物 8 对棒头草靶标具有较高抑制活性, 抑制率基本在 $90 \% \sim 100 \%$ 之间. 当以棒头草为靶标时, 通过对 $8 \mathrm{~g}, 8 \mathrm{~h}, 8 \mathrm{i}, 8 \mathrm{j}$ 的抑制活性比较可以发现, 氯取代 的目标化合物的抑制活性高于氟取代的目标化合物, 为 探究和优化该类化合物提供了思路.

\section{2 结论}

依据活性基才拼接原理, 在芳氧苯氧丙酸类结构基 础上引入酰胺结构, 并采取了生物酶拆分技术, 设计并 合成了 10 个新型 $\mathrm{R}$ 构型芳氧苯氧丙酰胺类化合物. 初 步的生测结果表明, 在 $150 \mathrm{~g} / \mathrm{ha}$ 剂量下, 目标化合物均 对单子叶禾本科杂草(䓟草、棒头草、早熟禾)显示出较 高的选择性除草活性. 尤其是在以棒头草为靶标时, 化 合物 8a, 8i, 8j 具有 $100 \%$ 防除效果. 这些实验数据为芳
氧苯氧丙酰胺类化合物的进一步优化奠定了基础.

\section{3 实验部分}

\section{1 仪器与试剂}

Bruker AVANC III $500 \mathrm{MHz}$ 核磁共振仪; 上海精密 科学仪器有限公司 X-4 型数字显示熔点测定仪(温度计 未校正); UPLC H-CLASS/QTOF G2-XS 质谱仪; 上海上 自仪转速表仪表电机有限公司 LOOYE ZX98-1 旋转蒸 发仪; 岛津 LC-20AT 高效液相色谱仪; 陆恒生物有限公 司 DG $160 \mathrm{pH}$ 计; BT00-50 M 兰格蠕动洜; 柱层析分离 纯化(柱层析硅胶为 $\mathrm{H}$ 型，青岛海洋化工厂，200～300 目). 所用试剂和溶剂均为分析纯.

\subsection{2-(4-羟基苯氧基)丙酸甲酯(2)的合成}

$\mathrm{N}_{2}$ 保护下, 在 $100 \mathrm{~mL}$ 的四口烧瓶中加入 $\mathrm{CH}_{3} \mathrm{OH}$ $(15 \mathrm{~mL}) 、 \mathrm{Na}_{2} \mathrm{SO}_{3}(0.003 \mathrm{~mol})$ 、对苯二酚 $(0.050 \mathrm{~mol})$, 升 温至 $30{ }^{\circ} \mathrm{C}$ ，缓慢滴加 $30 \% \mathrm{CH}_{3} \mathrm{ONa}$ 的甲醇溶液 $\left(\mathrm{CH}_{3} \mathrm{ONa} 0.075 \mathrm{~mol}\right)$. 滴毕保温 $0.5 \mathrm{~h}$ 后, 滴加 2-氯丙酸 甲酯 $(0.033 \mathrm{~mol})$, 反应约 6 7 h. 滴加浓盐酸调节 $\mathrm{pH}$ 至 $2 \sim 3$, 加入二氯甲烷萃取, 摚拌分层后分离出有机相, 再用饱和碳酸氢钠溶液洗涤 $(20 \mathrm{~mL} \times 3)$, 经无水硫酸钠 干燥后脱溶, 得到相应的中间体 $2^{[39]}$, 柱层析 $[V($ 石油 醚) $: V($ 乙酸乙酯 $)=1 ： 5$ ] 分离纯化得到红棕色液体 $4.562 \mathrm{~g}$, 产率 70.5\%. ${ }^{1} \mathrm{H}$ NMR (500 MHz, DMSO- $\left.d_{6}\right) \delta$ : $9.01(\mathrm{~s}, 1 \mathrm{H}), 6.72$ (d, $J=9.0 \mathrm{~Hz}, 2 \mathrm{H}), 6.67$ (d, $J=9.0 \mathrm{~Hz}$, 2H), $4.77(\mathrm{q}, \quad J=7.0 \mathrm{~Hz}, 1 \mathrm{H}), 3.66(\mathrm{~s}, 3 \mathrm{H}), 1.46(\mathrm{~d}, J=$ $7.0 \mathrm{~Hz}, 3 \mathrm{H})$.

\section{3 (R)-2-(4-羟基苯氧基)丙酸甲酯(3)的合成}

在 $500 \mathrm{~mL}$ 的四口烧瓶中加入中间体 $2(0.102 \mathrm{~mol})$ 和水 $(200 \mathrm{~mL})$, 搅拌并通过 $\mathrm{pH}$ 计-蠕动洜联用仪蠕动 
$0.5 \mathrm{~mol} / \mathrm{L}$ 的 $\mathrm{NaOH}$ 溶液调节四口烧瓶体系 $\mathrm{pH}$, 当反应 液 $\mathrm{pH}$ 为 7.0 时, 加入米曲霉 WZ007 (3 g), 控制体系温 度为 $35{ }^{\circ} \mathrm{C}$. 待 $\mathrm{pH}$ 计示数保持不变后, 停止反应, 减压 抽滤除去固体酶并用乙酸乙酯洗涤滤饼数次, 滤液用乙 酸乙酯萃取 $(50 \mathrm{~mL} \times 3)$ 并合并有机相, 经无水硫酸钠干 燥后, 旋蒸得到红棕色粘稠液体 $8.846 \mathrm{~g}$, 即得到中间体 (R)-2-(4-羟基苯氧基)丙酸甲酯(3) ${ }^{[40]}$, 收率 88.4\%. 经手 性液相色谱分析得中间体 3 的 $e e$ 值可达 $98.4 \%$. ${ }^{1} \mathrm{H}$ NMR (500 MHz, Chloroform- $d$ ) $\delta: 6.85 \sim 6.68(\mathrm{~m}, 4 \mathrm{H}), 5.48$ (s, $1 \mathrm{H}), 4.69$ (q, $J=6.5 \mathrm{~Hz}, 1 \mathrm{H}), 3.78$ (s, 3H), 1.61 (d, $J=6.5$ $\mathrm{Hz}, 3 \mathrm{H})$.

余下水相再用浓盐酸溶液调节 $\mathrm{pH}$ 至 $2 \sim 3$, 并用乙 酸乙酯萃取 $(50 \mathrm{~mL} \times 3)$, 经无水硫酸钠干燥后脱溶得到 白色固体 $8.031 \mathrm{~g}$, 即拆分副产物(S)-2-(4-羟基苯氧基)丙 酸(4), 收率为 $86.5 \%$. m.p. $139 \sim 141{ }^{\circ} \mathrm{C}$ ( Lit. $^{[41]}$ 139 $\left.140{ }^{\circ} \mathrm{C}\right) ;{ }^{1} \mathrm{H}$ NMR $\left(500 \mathrm{MHz}\right.$, DMSO- $\left.d_{6}\right) \delta: 12.85(\mathrm{~s}, 1 \mathrm{H})$, $8.96(\mathrm{~s}, 1 \mathrm{H}), 6.74 \sim 6.61(\mathrm{~m}, 4 \mathrm{H}), 4.63$ (q, $J=7.0 \mathrm{~Hz}, 1 \mathrm{H})$, $1.45(\mathrm{~d}, J=6.5 \mathrm{~Hz}, 3 \mathrm{H})$.

\subsection{2-(4-羟基苯氧基)丙酸(5)的合成}

在 $100 \mathrm{~mL}$ 三口烧瓶中加入(S)-2-(4-羟基苯氧基)丙 酸(4) $(0.006 \mathrm{~mol})$ 和甲醇 $(0.275 \mathrm{~mol})$, 插入干燥管, 搅拌 下加入 $98 \%$ 的浓硫酸 $(0.825 \mathrm{mmol})$, 加热回流反应约 $8 \mathrm{~h}$, 停止反应. 待反应液冷却, 用 $\mathrm{NaOH}$ 稀溶液调节溶液 $\mathrm{pH}$ 为 7 , 旋蒸回收溶剂甲醇. 用乙酸乙酯 $(15 \mathrm{~mL} \times 3)$ 萃取 脱溶得到浅棕色粘稠液体 $1.11 \mathrm{~g}$, 即产物 $(S)$-2-(4-差基 苯氧基)丙酸甲酯, 收率 95.7\%. 将所得 $(S)$-2-(4-差基苯 氧基)丙酸甲酯加入 $100 \mathrm{~mL}$ 三口烧瓶中, 并用甲苯(20 $\mathrm{mL}$ ) 溶解，继续加入 $30 \%$ 的 $\mathrm{CH}_{3} \mathrm{ONa}$ 的甲醇溶液 $\left(\mathrm{CH}_{3} \mathrm{ONa} 6.4 \mathrm{mmol}\right)$, 加热回流反应约 $14 \mathrm{~h}$ 停止. 脱溶, 加入水 $(30 \mathrm{~mL})$, 调节 $\mathrm{pH}$ 至 2 3, 抽滤处理到浅棕色固 体 $(R, S)$-2-(4-羟基苯氧基)丙酸 $(5) 0.953 \mathrm{~g}$, 收率 91.2\%. m.p. $142 \sim 144{ }^{\circ} \mathrm{C}$ (Lit. ${ }^{[42]} 144{ }^{\circ} \mathrm{C}$ ); $[\alpha]_{\mathrm{D}}^{20}-5.0$ (c 0.01 , $\mathrm{CH}_{3} \mathrm{OH}$ ); ${ }^{1} \mathrm{H}$ NMR (500 MHz, DMSO- $\left.d_{6}\right) \delta: 12.86(\mathrm{~s}, 1 \mathrm{H})$, $9.01(\mathrm{~s}, 7 \mathrm{H}), 6.76 \sim 6.59(\mathrm{~m}, 4 \mathrm{H}), 4.61(\mathrm{q}, J=6.5 \mathrm{~Hz}, 1 \mathrm{H})$, $1.43(\mathrm{~d}, J=6.5 \mathrm{~Hz}, 3 \mathrm{H})$.

\section{5 (R)-2-(4-羟基苯氧基)丙酸(6)的合成}

在 $100 \mathrm{~mL}$ 的三口烧瓶中加入中间体 $3(0.050 \mathrm{~mol})$, 边摚拌边缓慢倒入配制好的 $10 \%$ 的 $\mathrm{NaOH}(40 \mathrm{~mL})$ 溶液, 常温反应 $3 \mathrm{~h}$. 反应结束调节体系 $\mathrm{pH}$ 至 $2 \sim 3$, 用乙酸乙 酯萃取 $(40 \mathrm{~mL} \times 2)$, 合并有机相, 干燥、脱溶得浅棕色固 体中间体 $68.4 \mathrm{~g}$, 收率 $92.3 \%$. m.p. $139 \sim 141{ }^{\circ} \mathrm{C}$ (lit. ${ }^{[43]}$ $\left.141 \sim 145{ }^{\circ} \mathrm{C}\right) ;{ }^{1} \mathrm{H}$ NMR $\left(500 \mathrm{MHz}\right.$, DMSO- $\left.d_{6}\right) \delta: 6.77 \sim$ $6.53(\mathrm{~m}, 4 \mathrm{H}), 4.61(\mathrm{q}, J=6.5 \mathrm{~Hz}, 1 \mathrm{H}), 1.43(\mathrm{~d}, J=6.5 \mathrm{~Hz}$, $3 \mathrm{H})$.
3.6 (R)-2-\{4-[(5-氯-3-氟吡啶-2-基)氧基]苯氧基\}丙酸 (7)的合成

在装有恒压滴液漏斗、冷凝管和温度计的 $50 \mathrm{~mL}$ 三 口烧瓶中, 分别加入 DMF $(15 \mathrm{~mL})$ 、中间体 6 (0.035 $\mathrm{mol})$, 待完全溶解, 加入碳酸钾 $(0.053 \mathrm{~mol})$, 升温到 $70 \sim 80{ }^{\circ} \mathrm{C}$ 搅拌 $2 \mathrm{~h}$, 再在保温条件下用恒压滴液漏斗将 2,3-二氟-5-氯吡啶 $(0.032 \mathrm{~mol})$ 缓慢滴加至反应体系中. 然后升温至 $90{ }^{\circ} \mathrm{C}$, 反应约 7 $8 \mathrm{~h}$. 待反应液冷却, 冰浴 条件下调节体系 $\mathrm{pH}$ 至 $2 \sim 3$, 有米黄色固体析出, 水洗、 抽滤、干燥得米黄色固体 $9.087 \mathrm{~g}$, 即 $(R)-2-\{4-[(5$-氯-3氟吡啶-2-基)氧基]苯氧基 $\}$ 丙酸(7). 产率 91.3\%. m.p. $208 \sim 210{ }^{\circ} \mathrm{C}$ (Lit. ${ }^{[44]} 211 \sim 212{ }^{\circ} \mathrm{C}$ ); ${ }^{1} \mathrm{H}$ NMR $(500 \mathrm{MHz}$, DMSO- $\left.d_{6}\right) \delta: 13.09(\mathrm{~s}, 1 \mathrm{H}), 8.19(\mathrm{dd}, J=10.0,2.0 \mathrm{~Hz}$, $1 \mathrm{H}), 8.02(\mathrm{~d}, J=2.0 \mathrm{~Hz}, 1 \mathrm{H}), 7.17 \sim 7.06(\mathrm{~m}, 2 \mathrm{H}), 6.96 \sim$ $6.85(\mathrm{~m}, 2 \mathrm{H}), 4.83(\mathrm{q}, J=7.0 \mathrm{~Hz}, 1 \mathrm{H}), 1.51(\mathrm{~d}, J=7.0 \mathrm{~Hz}$, $3 \mathrm{H})$.

\section{7 目标化合物 8 的合成}

在 $50 \mathrm{~mL}$ 的三口烧瓶中先加入化合物 $7(2.0 \mathrm{mmol})$, 三乙胺 $(3.3 \mathrm{mmol})$ 和二氯甲烷 $(15 \mathrm{~mL})$, 待其溶解后, 冰 浴降温至 $5{ }^{\circ} \mathrm{C}$, 加入 $\mathrm{EDCI}(2.3 \mathrm{mmol})$, 滴加(取代)苯胺 (2.1 mmol), 滴加完毕后继续冰浴反应 $7 \sim 8 \mathrm{~h}$. 旋蒸除 去二氯甲烷, 经柱层析 $[V$ (石油醚) $: V$ (乙酸乙酯 $)=1$ : $3]$ 分离纯化得目标化合物 8 .

(R)-2-[4-(5-氯-3-氟-2-吡啶氧基)苯氧基]- $N$-苯基丙 酰胺 (8a): 白色固体, 产率 $67.3 \%, 82.3 \%$ ee. m.p. 111 $114{ }^{\circ} \mathrm{C} ;{ }^{1} \mathrm{H}$ NMR $\left(500 \mathrm{MHz}\right.$, DMSO- $\left.d_{6}\right) \delta: 10.15(\mathrm{~s}, 1 \mathrm{H})$, $8.18(\mathrm{dd}, J=9.5,2.0 \mathrm{~Hz}, 1 \mathrm{H}), 8.01(\mathrm{~d}, J=2.0 \mathrm{~Hz}, 1 \mathrm{H})$, $7.64(\mathrm{~d}, J=7.5 \mathrm{~Hz}, 2 \mathrm{H}), 7.32(\mathrm{t}, J=8.0 \mathrm{~Hz}, 2 \mathrm{H}), 7.15(\mathrm{~d}$, $J=9.0 \mathrm{~Hz}, 2 \mathrm{H}), 7.08(\mathrm{t}, J=7.5 \mathrm{~Hz}, 1 \mathrm{H}), 7.01$ (d, $J=9.0$ $\mathrm{Hz}, 2 \mathrm{H}), 4.88$ (q, $J=6.5 \mathrm{~Hz}, 1 \mathrm{H}), 1.57(\mathrm{~d}, J=6.5 \mathrm{~Hz}, 3 \mathrm{H})$; HRMS calcd for $\mathrm{C}_{20} \mathrm{H}_{17} \mathrm{ClFN}_{2} \mathrm{O}_{3}[\mathrm{M}+\mathrm{H}]^{+}$387.0906, found 387.0902 .

(R)-2-[4-(5-氯-3-氟-2-吡啶氧基)苯氧基]- $N$-(2-甲氧 基)苯基丙酰胺 $(8 \mathbf{b})$ : 白色固体，产率 66.8\%, 99.7\% ee. m.p. 77 $79{ }^{\circ} \mathrm{C} ;{ }^{1} \mathrm{H}$ NMR (500 MHz, DMSO- $\left.d_{6}\right) \delta: 9.27$ (s, 1H), $8.17(\mathrm{dd}, J=10.0,2.0 \mathrm{~Hz}, 1 \mathrm{H}), 8.04 \sim 7.98(\mathrm{~m}$, $2 \mathrm{H}), 7.17(\mathrm{~d}, J=9.0 \mathrm{~Hz}, 2 \mathrm{H}), 7.14 \sim 7.03(\mathrm{~m}, 4 \mathrm{H}), 6.93(\mathrm{t}$, $J=7.5 \mathrm{~Hz}, 1 \mathrm{H}), 5.06$ (q, $J=6.5 \mathrm{~Hz}, 1 \mathrm{H}), 3.83(\mathrm{~s}, 3 \mathrm{H}), 1.55$ (d, $J=7.0 \mathrm{~Hz}, 3 \mathrm{H}$ ); HRMS calcd for $\mathrm{C}_{21} \mathrm{H}_{19} \mathrm{ClFN}_{2} \mathrm{O}_{3}[\mathrm{M}+$ $\mathrm{H}]^{+}$417.1012, found 417.1002.

(R)-2-[4-(5-氯-3-氟-2-吡啶氧基)苯氧基]- $N$-(4-叔丁 基)苯基丙酰胺 $(\mathbf{8 c})$ ：土黄色固体，产率 69.5\%, 91.4\% ee. m.p. $100 \sim 107^{\circ} \mathrm{C} ;{ }^{1} \mathrm{H}$ NMR (500 MHz, DMSO- $\left.d_{6}\right) \delta$ : 10.10 (s, $1 \mathrm{H}), 8.17$ (dd, $J=10.0,2.0 \mathrm{~Hz}, 1 \mathrm{H}), 8.01$ (d, $J=$ $2.5 \mathrm{~Hz}, 1 \mathrm{H}), 7.54$ (d, $J=9.0 \mathrm{~Hz}, 2 \mathrm{H}), 7.32(\mathrm{~d}, J=9.0 \mathrm{~Hz}$, 
2H), $7.14(\mathrm{~d}, J=9.0 \mathrm{~Hz}, 2 \mathrm{H}), 7.00(\mathrm{~d}, J=9.0 \mathrm{~Hz}, 2 \mathrm{H}), 4.85$ (q, $J=6.5 \mathrm{~Hz}, 1 \mathrm{H}), 1.55(\mathrm{~d}, J=6.5 \mathrm{~Hz}, 3 \mathrm{H}), 1.25(\mathrm{~s}, 9 \mathrm{H})$; HRMS calcd for $\mathrm{C}_{24} \mathrm{H}_{25} \mathrm{ClFN}_{2} \mathrm{O}_{3}[\mathrm{M}+\mathrm{H}]^{+}$443.1532, found 443.1529 .

(R)-2-[4-(5-氯-3-氟-2-吡啶氧基)苯氧基]- $N$-(2,4-二 甲基)苯基丙酰胺(8d): 白色固体, 产率 73.4\%, 93.5\% ee. m.p. $119 \sim 121{ }^{\circ} \mathrm{C} ;{ }^{1} \mathrm{H}$ NMR (500 MHz, DMSO- $\left.d_{6}\right) \delta$ : 9.49 (s, 1H), 8.19 (dd, $J=10.0,2.0 \mathrm{~Hz}, 1 \mathrm{H}), 8.02$ (d, $J=$ $2.0 \mathrm{~Hz}, 1 \mathrm{H}), 7.19 \sim 7.10(\mathrm{~m}, 3 \mathrm{H}), 7.04(\mathrm{~d}, J=9.0 \mathrm{~Hz}, 3 \mathrm{H})$, $7.00 \sim 6.91(\mathrm{~m}, 1 \mathrm{H}), 4.91(\mathrm{q}, J=6.5 \mathrm{~Hz}, 1 \mathrm{H}), 2.25(\mathrm{~s}, 3 \mathrm{H})$, $2.06(\mathrm{~s}, 3 \mathrm{H}), 1.59$ (d, $J=6.5 \mathrm{~Hz}, 3 \mathrm{H})$; HRMS calcd for $\mathrm{C}_{22} \mathrm{H}_{21} \mathrm{ClFN}_{2} \mathrm{O}_{3}[\mathrm{M}+\mathrm{H}]^{+}$415.1219, found 415.1215.

(R)-2-[4-(5-氯-3-氟-2-吡啶氧基)苯氧基]- $N$-(2-甲基3 -氯)苯基丙酰胺 $(8 \mathrm{e})$ : 米黄色固体, 产率 $65.2 \%, 87.1 \%$ ee. m.p. $159 \sim 162{ }^{\circ} \mathrm{C} ;{ }^{1} \mathrm{H}$ NMR $\left(500 \mathrm{MHz}, \mathrm{DMSO}-d_{6}\right) \delta$ : $9.89(\mathrm{~s}, 1 \mathrm{H}), 8.20(\mathrm{dd}, J=10.0,2.0 \mathrm{~Hz}, 1 \mathrm{H}), 8.01$ (d, $J=$ $2.0 \mathrm{~Hz}, 1 \mathrm{H}), 7.32(\mathrm{dd}, J=7.5,2.0 \mathrm{~Hz}, 1 \mathrm{H}), 7.23 \sim 7.15(\mathrm{~m}$, 4H), 7.04 (d, $J=9.0 \mathrm{~Hz}, 2 \mathrm{H}), 4.95$ (q, $J=6.5 \mathrm{~Hz}, 1 \mathrm{H}), 2.12$ (s, 3H), $1.59(\mathrm{~d}, J=6.5 \mathrm{~Hz}, 3 \mathrm{H})$; HRMS calcd for $\mathrm{C}_{21} \mathrm{H}_{18} \mathrm{Cl}_{2} \mathrm{FN}_{2} \mathrm{O}_{3}[\mathrm{M}+\mathrm{H}]^{+}$435.0673, found 435.0670.

(R)-2-[4-(5-氯-3-氟-2-吡啶氧基)苯氧基]- $N$-(2-氟)苯 基丙酰胺 $(\mathbf{8 f})$ : 白色固体，产率 63.2\%，98.3\% ee. m.p. $73 \sim 75{ }^{\circ} \mathrm{C} ;{ }^{1} \mathrm{H}$ NMR (500 MHz, DMSO- $\left.d_{6}\right) \delta: 9.98(\mathrm{~s}$, $1 \mathrm{H}), 8.20(\mathrm{dd}, J=10.0,2.0 \mathrm{~Hz}, 1 \mathrm{H}), 8.02$ (d, $J=2.0 \mathrm{~Hz}$, $1 \mathrm{H}), 7.76(\mathrm{td}, J=8.0,2.0 \mathrm{~Hz}, 1 \mathrm{H}), 7.30 \sim 7.24(\mathrm{~m}, 1 \mathrm{H})$, $7.24 \sim 7.13(\mathrm{~m}, 4 \mathrm{H}), 7.02$ (d, $J=9.0 \mathrm{~Hz}, 2 \mathrm{H}), 5.03$ (q, $J=$ $6.5 \mathrm{~Hz}, 1 \mathrm{H}), 1.57(\mathrm{~d}, J=6.5 \mathrm{~Hz}, 3 \mathrm{H})$; HRMS calcd for $\mathrm{C}_{20} \mathrm{H}_{16} \mathrm{ClF}_{2} \mathrm{~N}_{2} \mathrm{O}_{3}[\mathrm{M}+\mathrm{H}]^{+}$405.0812, found 405.0807.

(R)-2-[4-(5-氯-3-氟-2-吡定氧基)苯氧基]- $N$-(4-氟)苯 基丙酰胺 $(8 \mathrm{~g})$ : 白色固体, 产率 $65.1 \%, 97.5 \%$ ee. m.p. $123 \sim 126{ }^{\circ} \mathrm{C} ;{ }^{1} \mathrm{H}$ NMR (500 MHz, DMSO- $\left.d_{6}\right) \delta: 10.23(\mathrm{~s}$, $1 \mathrm{H}), 8.19$ (dd, $J=10.0,2.0 \mathrm{~Hz}, 1 \mathrm{H}), 8.01$ (d, $J=2.5 \mathrm{~Hz}$, $1 \mathrm{H}), 7.65(\mathrm{dd}, J=9.0,5.0 \mathrm{~Hz}, 2 \mathrm{H}), 7.19 \sim 7.11(\mathrm{~m}, 5 \mathrm{H})$, $7.04 \sim 6.95(\mathrm{~m}, 3 \mathrm{H}), 4.86(\mathrm{q}, J=6.5 \mathrm{~Hz}, 1 \mathrm{H}), 1.55(\mathrm{~d}, J=$ $6.5 \mathrm{~Hz}, 3 \mathrm{H})$; HRMS calcd for $\mathrm{C}_{20} \mathrm{H}_{16} \mathrm{ClF}_{2} \mathrm{~N}_{2} \mathrm{O}_{3}[\mathrm{M}+\mathrm{H}]^{+}$ 405.0812, found 405.0808.

(R)-2-[4-(5-氯-3-氟-2-吡啶氧基)苯氧基]- $N$-(2,6-二 氟)苯基丙酰胺 $(8 \mathrm{~h})$ : 灰色固体, 产率 66.4\%, 96.9\% ee. m.p. 99 $102{ }^{\circ} \mathrm{C} ;{ }^{1} \mathrm{H}$ NMR (500 MHz, DMSO- $\left.d_{6}\right) \delta: 9.98$ (s, $1 \mathrm{H}), 8.18(\mathrm{dd}, J=10.0,2.0 \mathrm{~Hz}, 1 \mathrm{H}), 8.01$ (d, $J=2.0 \mathrm{~Hz}$, $1 \mathrm{H}), 7.67(\mathrm{td}, J=9.0,6.0 \mathrm{~Hz}, 1 \mathrm{H}), 7.36 \sim 7.30(\mathrm{~m}, 1 \mathrm{H})$, $7.18 \sim 7.12(\mathrm{~m}, 3 \mathrm{H}), 7.10 \sim 7.05(\mathrm{~m}, 1 \mathrm{H}), 7.04 \sim 6.97(\mathrm{~m}$, $3 \mathrm{H}), 4.98$ (q, $J=6.5 \mathrm{~Hz}, 1 \mathrm{H}), 1.55$ (d, $J=6.5 \mathrm{~Hz}, 3 \mathrm{H})$; HRMS calcd for $\mathrm{C}_{20} \mathrm{H}_{15} \mathrm{ClF}_{3} \mathrm{~N}_{2} \mathrm{O}_{3}[\mathrm{M}+\mathrm{H}]^{+}$423.0718, found 423.0712 .
(R)-2-[4-(5-氯-3-氟-2-吡啶氧基)苯氧基]- $N$-(3-氯)苯 基丙酰胺 $(8 \mathrm{i})$ ：白色固体，产率 70.7\%，94.5\% ee. m.p. $116 \sim 118{ }^{\circ} \mathrm{C} ;{ }^{1} \mathrm{H}$ NMR (500 MHz, DMSO- $\left.d_{6}\right) \delta: 10.38$ (s, $1 \mathrm{H}), 8.18(\mathrm{dd}, J=10.0,2.0 \mathrm{~Hz}, 1 \mathrm{H}), 8.01(\mathrm{~d}, J=2.0 \mathrm{~Hz}$, $1 \mathrm{H}), 7.85(\mathrm{t}, J=2.0 \mathrm{~Hz}, 1 \mathrm{H}), 7.57 \sim 7.53(\mathrm{~m}, 1 \mathrm{H}), 7.35(\mathrm{t}$, $J=8.0 \mathrm{~Hz}, 1 \mathrm{H}), 7.18 \sim 7.13(\mathrm{~m}, 3 \mathrm{H}), 7.00(\mathrm{~d}, J=9.0 \mathrm{~Hz}$, 2H), 4.88 (q, $J=6.5 \mathrm{~Hz}, 1 \mathrm{H}), 1.57$ (d, $J=6.5 \mathrm{~Hz}, 3 \mathrm{H})$; HRMS calcd for $\mathrm{C}_{20} \mathrm{H}_{16} \mathrm{Cl}_{2} \mathrm{FN}_{2} \mathrm{O}_{3}[\mathrm{M}+\mathrm{H}]^{+}$421.0517, found 421.0510 .

(R)-2-[4-(5-氯-3-氟-2-吡啶氧基)苯氧基]- $N$-(4-氯)苯 基丙酰胺 $(8 \mathbf{j})$ : 白色固体，产率 63.0\%, 96.6\% ee. m.p. $113 \sim 117{ }^{\circ} \mathrm{C} ;{ }^{1} \mathrm{H}$ NMR (500 MHz, DMSO- $\left.d_{6}\right) \delta: 10.30(\mathrm{~s}$, $1 \mathrm{H}), 8.18(\mathrm{dd}, J=10.0,2.0 \mathrm{~Hz}, 1 \mathrm{H}), 8.01(\mathrm{~d}, J=2.0 \mathrm{~Hz}$, 1H), 7.68 (d, $J=9.0 \mathrm{~Hz}, 2 \mathrm{H}), 7.38$ (d, $J=9.0 \mathrm{~Hz}, 2 \mathrm{H}), 7.15$ $(\mathrm{d}, J=9.0 \mathrm{~Hz}, 2 \mathrm{H}), 7.01(\mathrm{~d}, J=9.0 \mathrm{~Hz}, 2 \mathrm{H}), 4.88$ (q, $J=$ $6.5 \mathrm{~Hz}, 1 \mathrm{H}), 1.56(\mathrm{~d}, J=6.5 \mathrm{~Hz}, 3 \mathrm{H})$; HRMS calcd for $\mathrm{C}_{20} \mathrm{H}_{16} \mathrm{Cl}_{2} \mathrm{FN}_{2} \mathrm{O}_{3}[\mathrm{M}+\mathrm{H}]^{+} 421.0517$, found 421.0510 .

\section{8 除草活性测试}

温室盆栽测试在浙江省化工研究院国家南方农药 基地安评中心按照文献[45]方法进行，其除草活性结果 列于表 1 中.

致谢 感谢郑建永博士在生物酶拆分方面提供的技术 支持.

辅助材料(Supporting Information) 化合物 $8 \mathbf{a} \sim 8 \mathbf{j}$ 的 ${ }^{1} \mathrm{H}$ NMR 和 HRMS 图谱. 这些材料可以免费从本刊网站 (http://sioc-journal.cn/)上下载.

\section{References}

[1] Mu, J. X.; Shi, Y. X.; Yang, M. Y.; Sun, Z. H.; Liu, X. H.; Li, B. J.; Sun, N. B. Molecules. 2016, 21, 68.

[2] Liu, X. H.; Fang, Y. M.; Xie, F.; Zhang, R. R.; Shen, Z. H.; Tan, C. X.; Weng, J. Q.; Xu, T. M.; Huang, H. Y. Pest Manage. Sci. 2017, 73, 1900.

[3] Wang, H.; Zhai, Z. W.; Shi, Y. X.; Tan, C. X.; Weng, J. Q.; Han, L.; Li B. J.; Liu, X. H. Lett. Drug Des. Discovery 2019, DOI: 10 2174/1570180815666180704103047.

[4] Cheng, L.; Zhang, R. R.; Wu, H. K.; Xu, T. M.; Liu, X. H. Front. Chem. Sci. Eng. 2019, 13, 369.

[5] Zhou, J. H.; Dai, H.; Qian, H. W.; Du, X. C,; Mao, X. Y.; Shi, Y. J.; Feng, H.; Shi, J.; Yao, Y. Chin. J. Org. Chem. 2018, 38, 2122 (in Chinese).

(周家华，戴红，钱宏炜，杜显超，茅心宇，石玉军，冯浩，石健， 姚勇, 有机化学, 2018, 38, 2122.)

[6] Liu, X. H.; Xu, X. Y.; Tan, C. X.; Weng, J. Q.; Xin, J. H.; Chen, J. Pest Manage. Sci. 2015, 71, 292.

[7] Liu, X. H.; Zhai, Z. W.; Xu, X. Y.; Yang, M. Y.; Sun, Z. H.; Weng, J. Q.; Tan, C. X.; Chen, J. Bioorg. Med. Chem. Lett. 2015, 25, 5524.

[8] Cheng, L.; Zhao, W.; Shen, Z. H.; Xu, T. M.; Wu, H. K.; Peng, W. L.; Liu, X. H. Lett. Drug Des. Discovery 2019, 16, 29.

[9] Fang, Y. M.; Zhang, R. R.; Shen, Z. H.; Tan, C. X.; Weng, J. Q.; Xu, T. M.; Liu, X. H. Huang, H. Y.; Wu, H. K. Lett. Drug Des. 
Discovery 2018, 15, 1314.

[10] Liu, X. H.; Wang, Q.; Sun, Z. H.; Wedge, D. E.; Becnel, J.-J.; Estep, A. S.; Tan, C. X.; Weng, J. Q. Pest Manage. Sci. 2017, 73, 953.

[11] Shen, Z. H.; Sun, Z. H.; Becnel, J.-J.; Estep, A.; Wedge, D. E.; Tan, C. X.; Weng, J. Q.; Han, L, Liu. X. H. Lett. Drug Des. Discovery 2018, 15,951

[12] Shi, Y. J.; Zhou, Q.; Wang, Y.; Qian, H. W.; Ye, Y. L.; Feng, X.; Chen, H.; Li, Y. T.; Dai, H.; Wei, Z. H.; Wu, J. M. Chin. J. Org. Chem. 2018, 38, 2450 (in Chinese).

(石玉军, 周钱, 王杨, 钱宏炜, 叶玉林, 冯霞, 陈辉, 李雅婷, 戴红, 魏中昊, 吴锦明, 有机化学, 2018, 38, 2450.)

[13] Li, Z. F.; Luo, F. Y. Chin. J. Org. Chem. 2001, 21, 317 (in Chinese). (李再峰, 罗富英, 有机化学, 2001, 21,317.)

[14] Bao, W. J.; Wu, Y. G.; Mao, C. H.; Chen, M.; Huang, M. Z. Fine Chem. Intermed. 2007, 37, 1009 (in Chinese).

(包文娟, 吴永果, 毛春晖, 陈明, 黄明智, 精细化工中间体, 2007, 37, 1009.)

[15] Rendina, A. R.; Felts, J. M.; Beaudoin, J. D.; Craig-Kennard, A. C.; Look, L.-L.; Paraskos, S. L.; Hagenah, J. A. Arch. Biochem. Biophys. 1988, 265, 219.

[16] Smith, A. E. J. Agric. Food Chem. 1977, 25, 893.

[17] Walker, K. A.; Ridley, S. M.; Harwood, J. L. Biochem. J. 1988, $254,811$.

[18] Song, L. Y.; Hua, R. M.; Zhao, Y. C. J. Hazard. Mater. 2005, 118, 247.

[19] Zhao, W.; Xing, J. H.; Xu, T. M.; Peng, W. L.; Liu, X. H. Front. Chem. Sci. Eng. 2017, 11, 363

[20] Zhao, W.; Shen, Z. H.; Xu, T. M.; Peng, W. L.; Liu, X. H. J. Heterocycl. Chem. 2017, 54, 1751.

[21] Cheng, L.; Shen, Z. H.; Xu, T. M.; Tan, C. X.; Weng, J. Q.; Han, L.; Peng, W. L.; Liu. X. H. J. Heterocycl. Chem. 2018, 55, 946.

[22] Zhao, W.; Shen, Z. H.; Xing, J. H.; Yang, G.; Xu, T. M.; Peng, W. L.; Liu, X. H. Chem. Pap. 2017, 71, 921.

[23] Zhao, W.; Shen, Z. H.; Xing, J. H.; Xu, T. M.; Peng, W. L.; Liu, X. H. Chin. J. Struct. Chem. 2017, 36, 423.

[24] Liu, X. H.; Qiao, L.; Zhai, Z. W.; Cai, P.-P.; Cantrell, C. L.; Tan, C. X.; Weng, J. Q.; Han, L.; Wu, H. K. Pest Manage. Sci. 2019, 75, 2892.

[25] Sun, N. B.; Shi, Y. X.; Liu, X. H.; Ma, Y.; Tan, C. X.; Weng, J. Q.; Jin, J. Z.; Li, B. J. Int. J. Mol. Sci. 2013, 14, 21741.

[26] Zhang, J, H.; Niu, L. Z.; Li, Y.; Liu, S.; Jiang, L. Chin. J. Org. Chem. 2018, 38, 1842 (in Chinese).
(张君辉, 牛李智, 李映, 刘思, 姜林, 有机化学, 2018, 38, 1842.)

[27] Liu, X. H.; Weng, J. Q.; Wang, B. L.; Li, Y. H.; Tan, C. X.; Li, Z. M. Res. Chem. Intermed. 2014, 40, 2605.

[28] Che, C. L.; Hu, Y. M.; Ding, S.-S.; Xiao, Y. M.; Li, J. Q.; Qin, Z. H. Chin. J. Org. Chem. 2019, 39, 419 (in Chinese) (车传亮, 胡益敏, 丁珊珊, 肖玉梅, 李佳奇, 覃兆海, 有机化学, 2019, 39, 419.)

[29] Moon, J. K.; Keum, Y. S.; Hwang, E. C.; Park, B. S.; Chang, H. R.; Li, Q. X.; Kim, J. H. J. Agric. Food Chem. 2007, 55, 5416.

[30] Yu, Z. H.; Shi, D. Q. Phosphorus, Sulfur Silicon Relat. Elem. 2010, $185,2316$.

[31] Liu, X. H.; Zhao, W.; Shen, Z. H.; Xing, J. H.; Yuan, J.; Yang, G.; Xu, T. M.; Peng, W. L. Bioorg. Med. Chem. Lett. 2016, 26, 3626.

[32] Asad, M. A. U.; Lavoie, M.; Song, H.; Jin, Y. J.; Fu, Z. W.; Qian, H. F. Sci. Total Environ. 2017, 580, 1287.

[33] Liu, X. H.; Zhao, W.; Shen, Z. H.; Xing, J. H.; Xu, T. M.; Peng, W. L. Eur. J. Med. Chem. 2017, 125, 881.

[34] Schurter, R. US 4713109, 1988 [Chem. Abstr. 1988, 109, 128841].

[35] Kim, D. W. WO 2000005956, 2000 [Chem. Abstr. 2000, 132, 118794].

[36] Zheng, J. Y.; Wu, J. Y.; Zhang, Y. J.; Wang, Z. J. Mol. Catal. B: Enzym. 2013, 97, 62 .

[37] Kourist, R.; Pablo, D. D. M.; Miyamoto, K. Green Chem. 2011, 13, 2607.

[38] Erdemir, S.; Yilmaz, M. J. Inclusion Phenom. Macrocyclic Chem. 2012, 72, 189

[39] Telschow, J. E. US 4614814, 1989 [Chem. Abstr. 1989, 111, 57259].

[40] Han, C. P.; Sun, J.; Huang, T. H. Chem. Reag. 2015, 37, 354 (in Chinese). (韩翠平, 崔杰, 黄统辉, 化学试剂, 2015, 37, 354.)

[41] Zhang, P. F.; Zhong, H.; Villain, F. US 20050240049, 2005 [Chem. Abstr. 2005, 143, 422130].

[42] Sobotka, H.; Austin, J. J. Am. Chem. Soc. 1952, 74, 3813.

[43] Shen, Y. C.; Li, Y. X.; Wang, P. Chin. J. Synth. Chem. 2006, 14, 398 (in Chinese).

(申永存, 李燕霞, 王平, 合成化学, 2006, 14, 398.)

[44] Sugata, R.; Shashi, B. S. J. Environ. Sci. Health, Part B 2005, 40, 525.

[45] Sun, Z. H.; Huang, W.; Gong, Y.-Y.; Lan, J.; Liu, X. H.; Weng, J. Q.; Li, Y. S.; Tan, C. X. Chin. J. Org. Chem. 2013, 33, 2612 (in Chinese).

(孙召惠, 黄伟, 贡云芸, 蓝健, 刘幸海, 翁建全, 李永曙, 谭成 侠，有机化学，2013，33，2612.) 